\title{
Privacy and the mashrabiya screen: Knowledge is Sweeter than Honey
}

\author{
LISA DICKSON
}

Kent Law School

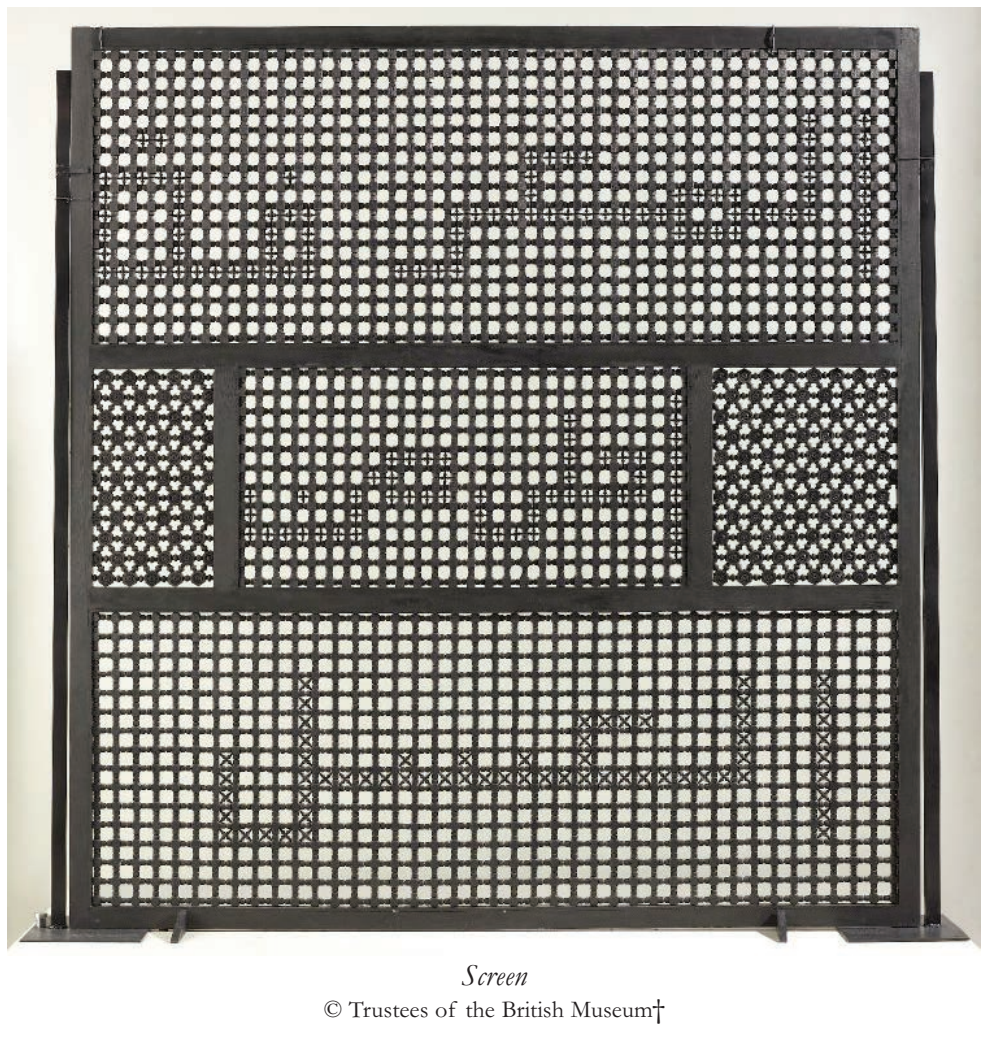

\begin{abstract}
$\underline{\text { Abstract }}$
This paper is offered to demonstrate the value of legal objects in the consideration of key legal concepts. In it I indicate the opportunities presented by an encounter with Susan Hefuna's large Mashrabiya Screen artwork in the British Museum to supplement, criticise and disrupt current thinking and attitudes towards the concept of privacy. In contrast to the increasingly contested and transactional nature of contemporary understandings of this concept, in which privacy is sometimes imagined just as one more complex function in the reasonable management of dataflows, Hefuna's screen can belp to articulate and support a different approach to privacy. This approach is Privacy by Design, and through a consideration of the physicality of Hefuna's work, together with her own artistic ambition, my claim is that her art object helps to make the alternative approach to privacy manifest and tangible, prompting a reappraisal of the proper scope and nature of privacy protection.
\end{abstract}

Keywords: privacy; Privacy by Design; data flows; screens; Susan Hefuna.

$\dagger \quad<$ www.britishmuseum.org/research/collection_online/collection_object_details.aspx?objectId=3153911 \&partId $=1>$ 


\section{Introduction}

$\mathrm{I}_{\mathrm{r}}^{\mathrm{n}}$ this brief paper my general aim is simple: to demonstrate some of the ways in which reflection on a single object can help to articulate and reappraise a topic of current interest in legal scholarship. That topic is privacy, and the object is a physical mashrabiya screen created by the artist Susan Hefuna, ${ }^{1}$ held in the British Museum and entitled Knowledge is Sweeter than Honey.

In some ways the links between object and topic are obvious and perhaps even appear trivial. It is obvious and intuitive, for example, that screens promote privacy, precisely because you cannot easily see through them. It also seems obvious that screens hinder privacy because a hidden observer can peer out at unsuspecting activity from beyond their cover. However, law and legal scholarship have recently played a primary role in the marginalisation of robust and intuitive understandings of privacy such as those in play here. Instead, in multiple jurisdictions and through multiple legal cases and instruments, the notion has become nebulous and gradable, 'a difficult and complex concept' whose instances are difficult to identify, to be found either broken down into myriad types and kinds, or cast simply as another transaction cost or marker in the algorithms that govern complex data flows. My claim is that Hefuna's screen physically reminds us of the intuitive absolutes that until recently were taken to characterise the concept and which are now in danger of being left behind. In fact, the specific jurisprudential aim of this paper is to show how Hefuna's screen helps articulate a different approach to privacy in law, in which privacy is not a rather shapeless concern in the reasonable management of data flows, but is a robust value that needs to be 'baked into' 2 the hardware and technology that people actually use. In short, this paper uses Hefuna's screen in support of 'Privacy by Design'.

A few qualifications are needed before my essay can begin. First, I make no distinction in what follows between matters that bear on pedagogy (i.e. using Hefuna's screen to promote student understanding and reflection on privacy) and scholarship (i.e. the production of new academic research on privacy and related matters). This is because the insight and opportunity offered by the reflection in question are of value to both, as I hope will become apparent as discussion proceeds. As Hannan et al observe, 'facing students with an unknown object and asking them to deduce what they can from its physical form, encourages just the sort of analysing and hypothesizing that are the life forces of scholarly enquiry'. ${ }^{3}$ This is precisely my methodological ambition in putting Hefuna's screen to use and holds as much for academic researchers as for student learning. Indeed, underpinning my approach is a single thesis, as applicable to scholarship as to learning and teaching. The thesis is that asking familiar questions (here, about the concept of privacy in law) in the context of an object-instance (a wooden art object in a gallery in the British Museum) allows a development of ideas that is not readily available in more typical treatments of privacy either through metaphysical generalities (so,

1 For bibliographic information on the artist - Susan Hefuna - see <wwwsusanhefuna.com>.

2 The phrase 'baked into' and similar is often used in discussion of privacy by design to suggest the need to ensure privacy settings are embedded into the process or product that they are 'so deeply baked-in they cannot be turned off'. See A Cavoukian and J Jonas, Privacy by Design in the Age of Big Data (Information and Privacy Commissioner of Ontario, Canada 2012) 2.

3 L Hannan, R Duhs and H Chatterjee, 'Object-based Learning: A Powerful Pedagogy for Higher Education', in Jos Boys, Museums and Higher Education Working Together: Challenges and Opportunities (Routledge 2016) 165. 
theorising the concept of privacy in the abstract), ${ }^{4}$ or through the routine sites for its application (thus, the textbook cases and other sites of its legal contestation). ${ }^{5}$ In this way I aim to use both Hefuna's screen to urge a pause in the current theorising (away) of privacy in law, and also to use the platform of privacy in law to press the merit of using museum and other objects to analyse and hypothesise in the legal arena, exactly as Hannan et al propose. I take both of these aims together as the discussion proceeds.

My argument is set out over the following three sections, each offered, fittingly enough, as a 'screen'. I want to call screens to mind throughout, which is one thing that an encounter with Hefuna's screen naturally presses on an observer. Each screen works in just the way Hefuna's does in the British Museum - both presented as an object in its own right, but also as a fixed point of mediation through which further ideas can be glimpsed, linked and brought into focus. In this way I set out a procession of ideas, each tethered to and prompted by the physical screen in question. So, the first screen presents the object itself. The second screen is brought into focus - is prompted immediately - by our encounter with the first and considers Hefuna's artistic intention in creating the object: ideas familiar to legal scholarship begin to intrude here. The third and final screen then moves through the first and second to interrogate the force of the screen for legal pedagogy and scholarship, with the focus resting entirely on the presently vexed concept of privacy. There then follows a brief conclusion.

Finally, a cautionary note is needed. My claim is not that the ideas on offer can only be found through consideration of Hefuna's artwork, nor is the claim that they are impossible to establish through more traditional scholarship. I do not claim that Hefuna's screen is a uniquely well-placed art object - even within the British Museum - for the delivery of the theoretical gains I have in mind. Rather my claim is simply that her screen, on a tour of the museum, can be used powerfully in the way I present. This, of course, is a strength rather than a weakness in regard to my general support for the use of museum artefacts for theoretical purposes. However, it would be a mistake to imagine from these caveats that the approach I set out is just a colourful tangent or adjunct to more worthwhile scholarship. We shall see that the method of engaging with Hefuna's physical object broadens the scope and reach of existing analytic approaches to privacy, in turn enabling a test and critique of the fitness for purpose of existing legal understandings of that concept.

\section{Screen 1: object}

The object in question is a work by the German-Egyptian artist Susan Hefuna. It is a wooden mashrabiya screen of five panels, of which three contain words written in Arabic. Created in 2007, the work was acquired by the British Museum in 2008 and is but one instance in a series of works by Hefuna - other physical screens, photographs - that take the mashrabiya screen as their subject. The screen, then, is a representation of an Egyptian 'mashrabiya' which has its roots in the Arabic phrase for 'cooling the water: such screens are a well-known feature of North African and Arabic architecture'. Although their history can be traced further back than the fourteenth century, these

4 For interesting discussion, see, for example, Daniel Solove. 'A Taxonomy of Privacy' (2005) 154 University of Pennsylvania Law Review 477; and R L Finn, D Wright and M Friedewald, 'Seven Types of Privacy' in European Data Protection: Coming of Age (Springer Netherlands 2013) 3-32.

5 See, for example, Murray v Express Newspapers and Another [2007] EWHC 1908 (Ch).

6 An interesting history of the development of the mashrabiya in Egyptian architectural culture is provided in J Mohamed, The Traditional Arts and Crafts of Turnery or Mashrabiya (Doctoral dissertation, Rutgers UniversityCamden Graduate School 2015). 
screens became a notable architectural feature in Cairo during this period, becoming part of protruding oriel windows, usually on the upper floors of houses, designed to catch any breezes to cool pots of water behind them and, as the mashrabiyas became more ornate and large, designed to protect the privacy of the people ${ }^{7}$ inside. Thus, as Feeny observes in a significant note:

Later, as they were fitted with cushioned beds running the length and breadth of the screen, they became comfortable havens in which the occupants could recline in cool privacy while gazing down at the streets or courtyards below and, unseen and unheard, share in the life of the outside world. ${ }^{8}$

Knowledge is Sweeter than Honey is just one of Hefuna's artistic renderings of such a screen.

Of the particular work itself the British Museum says this:

Knowledge is sweeter than honey: Sculpture, window-screen (mashrabiya), made of wood stained with black ink. Screen has outer frame and is divided internally into five 'windows'. Composed of numerous lathe-turned elements glued together to form geometric patterns. Further smaller elements are set within the three largest windows to form words in Arabic script.

The screen can be viewed by any of the visitors to the lower ground floor of the Sainsbury's Galleries located within the museum. As an art object it is striking - located within the African section, it is an imposing and freestanding piece that measures just over two metres wide and two metres tall, easily one of the larger objects in the gallery in which it resides. Its apparently 1:1 ratio with the mashrabiya screens whose form it appropriates naturally disturbs the borders of art and artefact - if you needed such a screen for a house you could quite easily adopt Hefuna's for the purpose. Indeed, those unfamiliar with mashrabiya screens may wonder at the relation between source and art object, between art and craft, and between facsimile and fake. Visitors to the gallery might begin to wonder whether the art in Hefuna's creation lies just in the insertion of the Arabic text, the rest of the object perhaps remaining unremarkably standard in form and construction. For now it is enough to imagine the bulk and heft of the piece and to realise that the photograph in the British Museum collection cannot do justice to its physical impact.

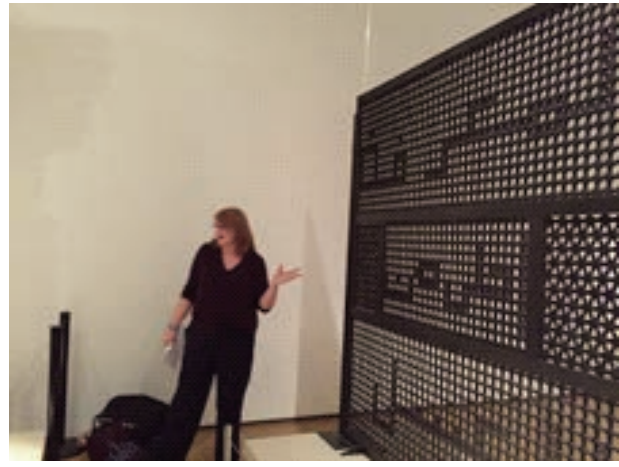

Figure 1: Hefuna's mashrabiya screen Photo by Amanda Perry-Kessaris

As can be seen in Figure 1, Hefuna's screen has considerable physical presence, not least because of its blackness in an otherwise light-coloured and well-lit setting. Interestingly, unlike many of the pieces located within this particular gallery, it is not enclosed in glass. The large screen stands on a base away from the gallery walls and is protected by short ropes, thus allowing observers to walk around the whole piece unimpeded by any casing. The ability to view the screen from both sides and, indeed, walk all around it allows the observer to break free of - or

7 Thoughtful discussion of the use of privacy within the construction of Muslim homes and architecture is provided in Z Othman, R Aird and L Buys, 'Privacy, Modesty, Hospitality, and the Design of Muslim Homes: A Literature Review' (2015) 4(1) Frontiers of Architectural Research 12-23.

8 John Feeney commentary in Saudi Aramco World (July/August 1974 print edition) 32-6, 33. 
problematise - consideration of which side is the 'inside' and which the 'outside'. 9 This question is not answered, of course, by the script in the three windows, for the question then becomes for whom - on which side - is the script written. In any case, many visitors will likely be unable to identify the side from which the Arabic writing reads intelligibly. That script resonates with the following lines, taken from Proverbs 24:

My son, eat thou honey, because it is good; and the honeycomb, which is sweet to thy taste: So shall the knowledge of wisdom be unto thy soul: when thou hast found it, then there shall be a reward, and thy expectation shall not be cut off. ${ }^{10}$

Of course, much can be made of Hefuna's part use of this Proverb in her artwork, but I do not aim to pursue these ideas in the current paper: for present purposes my discussion develops around an encounter with the sheer physicality of Hefuna's screen.

\section{Screen 2: Hefuna's artistic ambition}

In considering Hefuna's artistic intention we are pressed to attend to all the various dimensions of the physical object that, as it happens, Hefuna wants us to call to mind. Hence we must consider the screen from one side, then the other, and finally as an object itself. Hefuna has said this of her work with the mashrabiya screen:

In my experience, most human beings are not able to see the world without a screen of social and cultural projections. I discovered the shape of Mashrabiya screens - windows, blinds, various cultural forms of architectural elements - in Cairo. The Mashrabiya protects the inside world from the outside; filtering the light and cooling the inside space; allowing one to observe without being seen. For me the Mashrabiya became an abstract symbol that operates in two directions with the possibility for dialogue, rather than closure. It separates, yet also filters and joins. It signifies the 'in-between-ness' of being in two cultures at the same time that it reflects personal experiences dealing with cross-cultural codes. ${ }^{11}$

Here we can see the elements that enable the screen to be used so effectively as spur to legal pedagogy and research on privacy. This is because privacy, protecting 'the inside world from the outside' and 'allowing one to observe without being seen', is also bound together with other concerns and interests - it 'separates but also filters and joins' and 'operates in two directions'. As we shall see, in this way Hefuna's screen begins to present us with a context for privacy in which many of the contemporary approaches to that concept are focused and articulated.

Moreover, in presenting us with a mashrabiya screen Hefuna consciously evokes and continues a theme in North African art itself, in which such screens were 'rediscovered by Hassan Fathy in the 1980s'. Influenced as she and a generation of artists have been by Fathy, ${ }^{12}$ Hefuna's screen can be read as a continuing discourse on the mashrabiya screen and Egyptian culture - in which these screens have been culturally rehabilitated following their association with harems in Cairo. ${ }^{13}$ Moving beyond Fathy, Hefuna tells us that her deployment of the screen is an abstract symbol for her own (and others') cultural 'in-betweenness', allowing a 'possibility of dialogue' rather than just to bleakly include and exclude.

9 See Figure 2 below.

10 King James Bible, Proverbs 24:13-14.

11 Bettina Mathes, 'Interview with Susan Hefuna' (Flash Art 12 November 2010) <www.flashartonline.com/ 2010/11/tell-me-what-you-draw-and-i-will-tell-you-who-you-are-interview-with-susan-hefuna >.

12 See Hassan Fathy, Natural Energy and Vernacular Architecture (UN University 1986).

13 For further discussion see Mohamed (n 6). 
These various links and associations have not gone unnoticed by art critics and cultural historians. In a catalogue for an exhibition of Hefuna's work the significance of standpoint and context for Hefuna's mashrabiya are also picked out as significant:

Like a second skin, the Mashrabiya protects what is often most endangered in the encounter with the Other: the recognition of the differences that unite us. Mashrabiya: a shutter that doesn't expose. ${ }^{14}$

These remarks are arresting, particularly when set against contemporary technical (including legal) treatments of privacy in which the increasingly transactional deployment of the notion is strangely at odds with its value as 'a second skin' or 'shutter that doesn't expose'. But in a more nuanced assessment Nat Muller writes of Hefuna's creation:

Place a mashrabiya, in which words and phrases in German, Arabic and English are incorporated, in a gallery space and the one-way vision of inside looking out is scrambled and codes of what is private and public become confused. The vantage point of the viewer, both with regard to positioning in the actual space and to geo-ethnic context, becomes central to the work. ${ }^{15}$

Hefuna herself has recognised (and is interested in) the varied responses to her work that have thus resulted from the 'in-betweenness' and scrambled coding that her work evokes. She comments that:

I'm aware that people read my work differently, depending on their own cultural or social context. They only see what they know. In my experience, most human beings are not able to see the world without a screen of social and cultural projections.

However, in a typical note of hopefulness, Hefuna does not read these dynamics as necessarily negative, recalling again that the 'mashrabiya became a symbol that operates in two directions with the possibility for dialogue and awareness'. ${ }^{16}$

It is a notable feature of her study through mashrabiya that Hefuna intends both a projection of her own position of cultural in-betweenness through the screen, but also intends to call to mind and stimulate more universal themes of privacy, closure, mediation, context and dialogue. At times, even she has been surprised by the significance of context for the viewer. Speaking of differing responses to an earlier work she observes that, in Cairo:

One of my digital photographs of a Mashrabiya screen was instantly perceived as a familiar object. By contrast, all Western audiences had associated it with the Western concept of abstract art. This first-hand and unexpected feedback from Egypt was a complete surprise to me. A different audience saw the essence of the work and not its reflection, without having read any of my intentions or knowing anything about my background. From then on, my work was somehow enriched by this dual feedback: the historical, scientific, and aesthetic context of the work perceived by a Western eye, and the references that were immediately related to familiar surroundings by Egyptians. The reading of the work depended on the codes of each culture, the same form could refer to different ideas and images from the past and the present. ${ }^{17}$

14 'Knowledge Is Sweeter than Honey' (Catalogue Essay for Exhibition: Hefuna @ Vienna 2010, Galerie Grita Insam 2010).

15 Nat Muller, 'Making Things Permeable: Susan Hefuna' (2015) - interview available at $<$ http://susanhefuna.com/biography/43/press/>.

16 Alex Greenberger, 'Susan Hefuna on Mingling East and West in her Art' (Interview: Art Space 17 September 2013) <www.artspace.com/magazine/interviews_features/artist_to_watch/susan_hefuna-51606>.

17 Mathes (n 11). 
Nat Muller, again, thus records the impact of Hefuna's mature work, of which Knowledge is Sweeter than Honey is a prominent piece:

In her work Susan Hefuna masterfully turns inside into outside and vice versa. What often remains unanswered is what exactly constitutes this inside and outside, where it is located, and whose inside and outside it is to start with. The openness to these questions is precisely what makes Hefuna's practice so intriguing and multi-faceted from a conceptual and a formalist perspective. ${ }^{18}$

In Muller's summing-up we can already perceive the potential that Hefuna's screen presents for pedagogy and research on the concept of (and so the instances of) privacy in law. Thus we move through a physical object itself (Screen 1) and through Hefuna's ambition (Screen 2) to appreciate that her piece acts, and is intended to act, as a prompt to reflection on the shifting and multilayered dynamics of screens, in which closure, privacy, inclusion and exclusion become in the same instant meeting-points, filters, points of mediation and opportunities for dialogue. For Hefuna herself, the

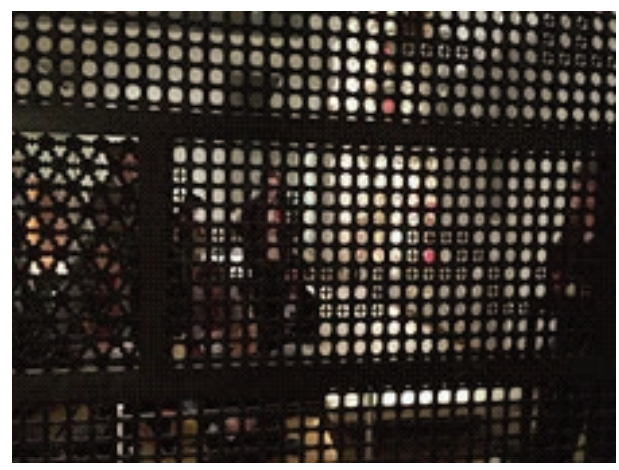

Figure 2: Inside and outside Photo by Amanda Perry-Kessaris mashrabiya presents a point of intersection spanning her divided cultural heritage. In short, her screen brings together many of the most significant elements of contemporary analysis of privacy in legal and other technical domains. At the very least, then, an encounter with her screen in the gallery begins to articulate and individuate ideas about privacy that run from simpler modal definitions ('a shutter that doesn't expose') to the complexities of context and contingency in which shifting standpoint is critical.

\section{Screen 3: privacy, law and the screen}

We are now in a position to use the artefact in Screen 1 and artistic ambition in Screen 2 to focus down on the legal conceptual issues that emerge in the encounter with Hefuna's mashrabiya screen art object. Here, the idea remains that her screen can act as a prompt to further reflection on the concept of privacy, or might even help to articulate that concept, or can open avenues for criticism and refinement of existing treatments and understandings of the notion.

One of the powerful ways in which Hefuna's art object helps to approach privacy is that it is sophisticated. Privacy might find a representation in other objects in the Museum - for example, a lock and key, ${ }^{19}$ or cabinet with secret panels, ${ }^{20}$ or an Egyptian sarcophagus. ${ }^{21}$ Knowledge is Sweeter than Honey is better placed than these, however, because - as we saw in the previous screen - her mashrabiya is both a material shutter and a point of intersection, of mediation and dialogue. For these reasons, it is particularly well suited to claim the attention of those for whom privacy and privacy problems appear (perhaps,

18 Muller (n 15).

19 For example, a Swedish padlock and key wrought from iron in the nineteenth century, BM Number 0402.4 $\mathrm{a}-\mathrm{b}$, not currently on display.

20 For example, the cabinet which is part of the Waddesdon Bequest, BM Number WB16, on display G2a/dc1.

21 For example, the Egyptian Sarcophagus from 600 BC found in the ancient Egypt and Sudan section, BM Number EA17, on display G4/B5. 
appear essentially) to concern the complexities of the negotiation over spaces and rights, of dataflow management and the balancing of legal and other interests. The legal context in which Hefuna's screen can intervene now needs to be set out with more clarity.

The main themes of contemporary legal approaches to privacy can be captured even within the limits of the present brief piece. Indeed, many of the relevant papers that have appeared over the last decade or so begin with useful and accurate summaries of the current scope of privacy analyses. Thus, we find running through the work of perhaps the principal contemporary theorist of privacy, Daniel Solove, readily available synopses of the different approaches on offer. ${ }^{22}$ Thus Solove has noted the prevailing mood among scholars that privacy is 'a concept in disarray. Nobody can articulate what it means ... Philosophers, legal theorists, and jurists have frequently lamented the great difficulty in reaching a satisfying conception of privacy. ${ }^{23}$ Opening his own account in 'Understanding Privacy', Solove cites Jonathan Franzen, who writes that 'on closer examination privacy proves to be the Cheshire cat of values: not much substance, but a very winning smile'. ${ }^{24}$ But Solove also makes careful note of the high value of privacy as recorded in a multitude of writings. These include that of Warren and Brandeis ${ }^{25}$ (privacy is 'the right most valued by civilised men'), Gavison (it is 'essential to democratic government'), Rachels (noting privacy is critical to 'our ability to create and maintain different sorts of social relationships with different people') and many others, whilst noting privacy's appearance in case law too - for example, in the US case, Lake $v$ Wal-mart Stores (privacy is 'integral to our humanity'). ${ }^{26}$ So there is plainly much at stake in the understanding of privacy for legal reasons alone, and this is the site for Robert Post's observation, often repeated by Solove himself, that:

Privacy is a value so complex, so entangled with competing and contradictory dimensions, so engorged with various and distinct meanings, that I sometimes despair whether it can usefully be addressed at all. ${ }^{27}$

Despite Post's despair, many scholars and courts have tried to make better sense of the concept, and Solove and others have done much to classify their attempts - usually with criticism in mind.

Solove's chief criticism with the scholarship in question is that it adopts what might be called an essentialist approach to defining privacy, in which theorists all search for some common thread running through privacy instances that might then be acclaimed a necessary marker or attribute of privacy itself. In Solove's analysis, the perceived essence has spanned (at least) the right to be let alone, limited access to the self, secrecy, control over personal information, personhood and intimacy. But, as Solove proclaims, these 'countless attempts' 28 have foundered on the inability to locate a common denominator, and this failure prompted Solove himself to argue for a different approach deriving from the philosophy of language of Ludwig Wittgenstein. ${ }^{29}$ In this approach we are urged to

22 Daniel J Solove, 'Conceptualizing Privacy' (2002) 90(4) California Law Review 1087; Daniel J Solove, Understanding Privacy (Harvard University Press 2008); Daniel J Solove, Notbing to Hide, The False Tradeoff between Privacy and Security (Yale University Press 2011).

23 Solove, Understanding Privacy (n 22) 1.

24 Ibid.

25 Samuel D Warren and Louis D Brandeis, 'The Right to Privacy' (1890) 4(5) Harvard 193.

26 See Solove, Understanding Privacy (n 22) ch 1.

27 Robert C Post, 'Three Concepts of Privacy' (2001) 89 Georgetown Law Journal 2087, 2087.

28 Solove, Understanding Privacy (n 22) 40.

29 See Ibid ch 3. 
give up the idea that meaning always depends upon necessary and sufficient definitional conditions and to embrace instead - at least for some concepts - the idea that meaning is more open-textured. For Solove, privacy is a 'cluster of many distinct and related things',30 'an umbrella term that refers to a wide and disparate group of related things', 31 and is to be theorised by finding 'a general framework to identify privacy harms or problems and to understand why they are problematic'. ${ }^{32}$ Solove's approach is different, then, because he conceptualises privacy 'from the bottom up', 33 and that 'a focal point for a theory of privacy should be the problems we want the law to address'. ${ }^{34}$ The difficulty for such a problem-based approach is that these problems seem exponentially on the rise.

Jon Mills expands on the phenomenon in his comment that 'the reasonable expectation of privacy recognized by the law does not keep pace with the varying types of information disclosure afforded by the rapidly advancing technologies such as the Internet, digitally recorded closed circuit television, and mobile communication devices'. 35 The increasing turnover of operating systems, privacy settings, new hardware and other sites in which privacy concerns might take hold (for example, online virtual reality) threaten to leave Solove's approach as unhelpful as the earlier approaches that draw on the classical theory of concepts. In fact, the confusing plurality of top-down 'cookie-cutter' essentialist definitions in Solove's line of fire is matched by the increasing plurality of privacy-problem instances in Solove's own treatment, with the resultant shapelessness of the notion an inevitable benefit to the technology companies, social media platforms, advertisers and so on for whom the acquisition of personal data has become vital. In fact, it is in this intensely practical arena, rather than in scholarly writing, that the most significant developments are to be found, and here it is possible to discern that privacy is increasingly construed as a function of data and information flow management.

Thus, the key terminology that often emerges in recent attempts to elucidate the concept of privacy is that of 'identifiable information' or 'personal data' - reducing the parameters of 'privacy' itself to that of dataflows. Indeed, in a recent co-authored piece (with Paul Schwarz), Solove notes the central importance, at least to the development of the legal concept, of personally identifiable information (PII), ${ }^{36}$ writing that: 'The scope of privacy laws typically turns on whether PII is involved.' 37 And this regulatory framework is hardly surprising given reliance on and widespread use of, amongst other things, digital devices. In social and media contexts we see concerns about 'privacy' related to social media, medical data and records, biometric identity cards and concern about the algorithms of 'big data' and the information being collected about individuals' web use and online preferences by companies and search engines. Indeed, the word 'screen' is now more familiar in the context of digital screens, and 'screening' is taken more often to relate to our use of such screens rather than to the activity of filtering out unwanted intrusion to protect our personal space. These changes have mostly proceeded imperceptibly and are little remarked upon in the relevant scholarly literature.

30 Ibid 40.

31 Ibid 41.

32 Ibid 49.

33 Ibid 49.

34 Ibid 75.

35 Jon L Mills, Privacy: The Lost Right (Oxford University Press 2009) 7.

36 P M Schwartz and D J Solove, 'The PII Problem: Privacy and a New Concept of Personally Identifiable Information' (2011) New York University Law Review 86, 1814. See 'Abstract' and particularly 1819.

37 Ibid 'Abstract'. 
The pragmatic reduction of privacy first to privacy problems or privacy harms and then to problems concerning information acquisition and management of dataflows is an understandable reaction to technological breakthrough and the inability of scholars to deliver a sharp-edged concept of privacy that can be put to work in legal settings. However, the perceived shapelessness of privacy that has resulted has allowed the notion to figure merely as a transaction cost in the creation of communication and information environments. This is precisely what two commentators have recently observed, Mulligan and King writing:

These plastic, sui generis, built environments give the companies that design them a privileged role in society. As architects of the 'playing fields' upon which individuals, and increasingly governments and private sector entities, engage, they can erect, alter, and obliterate structural barriers that afford or erode privacy through transaction costs ... Simply put, they have an unprecedented ability to reshape privacy norms on a global scale. ${ }^{38}$

It is in this vexed context of scholarship, pragmatism and technology that Hefuna's screen has the power to disrupt, acting as touchstone and motivation for a critical pedagogy and scholarly reflection on the notion of privacy itself. It has been effectively argued that, as we move to a more transparent society where the lines between 'private' and 'public' life are increasingly blurred, we place 'significant strain on existing privacy concepts and practices that depend on a boundary between private and public'. ${ }^{39}$ Hefuna's screen makes manifest the boundaries that are, still, possible. It is physical and architectural, standing for the mashrabiya screens that are built into the walls of houses in their vernacular setting. Hefuna's art object is large, undeniable, and calls to mind absolutes about privacy that are easily lost in the swirl of new technologies and algorithms of big data. It reminds us that screening can refer to the activity of protecting and securing providing a second skin or shutter that doesn't expose. To students who live in the contemporary, transactional information society it might appear to call back to a simpler time of absolutes now gone. But the power of Hefuna's screen is not merely that of historical artefact - sarcophagus for an earlier and simpler set of understandings - for it also stands as an emblem for a different approach to privacy that has emerged recently as a counter to the prevailing orthodoxy. This is the approach that is summed under the title privacy by design.

Privacy by design focuses on ensuring that privacy is a hardwired, default position in the systems and processes of platforms we use for our digital interactions. In this approach, privacy settings and positions must be considered throughout the design of a product or system, rather than being an add-on or patch at the end. Thus, 'Privacy by design is not a specific technology or product but a systematic approach to designing any technology that embeds privacy into the underlying specifications or architecture. 40

The idea, championed by Dr Ann Cavoukian, ${ }^{41}$ Information and Privacy Commissioner for Ontario, Canada, has gained support in recent years amongst

38 D K Mulligan and J King, 'Bridging the Gap between Privacy and Design' (2011) 14 University of Pennsylvania Journal of Constitutional Law 989-92.

39 M L Jones, 'Privacy without Screens and the Internet of Other People's Things' (2014) 51 Idaho Law Review $639,645$.

40 Ira S Rubinstein, 'Regulating Privacy by Design' (2011) 26(3) Berkeley Technology Law Journal 1409-56, 1412.

41 See A Cavoukian, 'Privacy by Design. Take the Challenge' (Information and Privacy Commissioner of Ontario, Canada, June 2009) or Privacy by Design website of the Information and Privacy Commissioner's Office available at <www.ipc.on.ca/wp-content/uploads/2013/09/pbd-primer.pdf>. 
information experts and practitioners. Indeed, internationally, data commissioners have summarised the approach in a resolution:

Knowing that with technological advances come new challenges to privacy and to the ability of individuals to exercise their information rights effectively; Accepting that existing regulation and policy alone are not sufficient fully to safeguard privacy; Recognizing that embedding privacy as the default into the design, operation and management of ICT and systems, across the entire information life cycle, is necessary to fully protect privacy. ${ }^{42}$

Perhaps even more significantly, privacy by design is about to become enshrined, in the European Union (EU) at least, in the newly created General Data Practice Regulations (GDPR) which will take direct effect throughout the EU in April 2018. ${ }^{43}$ Hefuna's screen plainly stands for privacy by design in a material sense, making manifest the nonnegotiable qualities of hardware-focused privacy protection. In this way the art object does not 'support' privacy by design in a simplistic sense, but rather helps students and researcher to bring into focus the full scope of complex issues that are in contention throughout the debate. After all, privacy that is 'baked into' IT and other hardware may not be attractive to individuals as consumers and certainly not to the companies and organisations for whom it would present a cost and impediment to their activity. So there are arguments still to be had. ${ }^{44}$ Instead, Hefuna's work, encountered in the museum, allows us to better appreciate the proper range and possibility of the concept of privacy itself by calling to mind protection through design - screening as second skin - that until recently could be taken for granted. The view I offer in this paper is that privacy by design is significant and welcome not least because, like Hefuna's screen, it reminds us that until recently we did not think of privacy as a relative concept (such as 'tallness') that depends just upon context for its correct extension, but rather as an absolute - though gradable concept (such as 'emptiness') that can be a closed value at one end. 45 These distinctions are not apparent in current academic literature, and the power of Hefuna's screen to remind us of them can be of real importance to contemporary theorising of the notion.

Hefuna's art work is also important in another way, already noted above, because of its multilayered sophistication that was evident in the discussion in Screen 2. Unlike other objects that might be used, Hefuna's mashrabiya screen, particularly as presented as art object in the museum, plays with and unsettles ideas of inside and outside, inclusion and exclusion, and the difficult negotiations of values of closure and dialogue, privacy and mediation. Significantly, however, the screen enables us to appreciate that, at least for some sites of privacy, these are debates that can run - indeed, can only run - because the central concept of privacy is already plainly to be seen, clearing the ground for productive

42 32nd International Conference of Data Protection and Privacy Commissioners, Jerusalem, Israel 27-29 October, 2010 Resolution on Privacy by Design <https://edps.europa.eu/sites/edp/files/publication/10_ 10-27_jerusalem_resolutionon_privacybydesign_en.pdf $>$.

43 Art 25(1) of the GDPR entitled 'Data Protection by Design and Default' states: 'Taking into account the state of the art, the cost of implementation and the nature, scope, context and purposes of processing as well as the risks of varying likelihood and severity for rights and freedoms of natural persons posed by the processing, the controller shall, both at the time of the determination of the means for processing and at the time of the processing itself, implement appropriate technical and organisational measures.' It remains to be seen how this will be achieved in the coming years and how much of a margin of appreciation will be left with the flexibility drawn into the provision by the opening phrase.

44 Solove has claimed that, while the development of privacy by design is to be welcomed, 'without a conception of privacy, Privacy by Design is like designing a building without a blueprint or baking a cupcake without a recipe' (Daniel Solove, (Privacy and Security Blog: Teach Privacy 14 July 2015) $<$ www.teachprivacy.com/privacy-by-design-4-key-points/>.

45 I owe this way of putting things to conversations with my colleague Dr Stephen Pethick. 
discussions about whether privacy is a good thing, or whether it is better to use screens to exclude than include, or to question who gets to afford such a screen - without miring these critical social and political questions in the perceived shapelessness of conceptual theorising and philosophy of language. Of course, Hefuna's artwork is not the only museum artefact that can work to such effect, but the opportunity it provides to prompt these thoughts (particularly, as I have found, standing with others in front of and behind it in the gallery) is considerable.

\section{Conclusion}

In sympathy with the insight offered by Perry-Kessaris in the introduction to this volume, Hefuna's mashrabiya aids legal enquiry by enabling us to visualise and make tangible a legal issue or problem that otherwise can appear abstract and esoteric. Hefuna's screen makes manifest issues of privacy. However, this is not simply a matter of making the concept tangible so that people can better grasp existing issues, for engagement with the mashrabiya allows us to go further, helping us to locate and refine our existing legal understandings, and so to better articulate or resolve the problems that have been felt in this area.

I noted in the introduction that, rather obviously, screens promote privacy, precisely because you cannot easily see through them, and hinder privacy, because a hidden observer can peer out at unsuspecting activity from beyond their cover. But though such robust intuitions seem trivial they nonetheless represent a perspective on privacy that is becoming marginalised at an increasing and, I think, worrying rate. Knowledge is sweeter than honey - certainly to many technology giants, advertisers, governmental organisations and social media platforms - and the present shapelessness of privacy as a concept has inevitably served their interests well. Against this drift, Hefuna's screen is a strong motif and reminder of the proper scope of the concept, whilst making manifest the idea of privacy by design. And through these things it might also, perhaps, help the case for rebalancing privacy interests in favour of citizens. 